\title{
La apertura al comercio exterior y sus efectos sobre la productividad en presencia de diferencias intersectoriales
}

\author{
Antonio Navas Ruiz ${ }^{\mathrm{a}}$
}

Universidad de Alicante

\section{RESUMEN}

Este artículo estudia los efectos que la apertura de una economía al comercio exterior, tiene sobre la productividad a nivel de empresa y a nivel de industria en un entorno donde existen diferencias intersectoriales en niveles de competencia en autarquía. Cuando la economía se abre al comercio internacional, aquellos sectores que son menos competitivos sufren mucho más la competencia extranjera, por lo cual reducen mucho más sus márgenes, y aumentan mucho más el volumen de producción y el personal dedicado a I+D, aumentando la productividad de sus empresas. En presencia de diferencias importantes en niveles de competencia inicial entre sectores, una apertura global puede generar una reducción del personal dedicado a $\mathrm{I}+\mathrm{D}$ en aquellos sectores más competitivos debido a un efecto crowding-out por parte de los otros sectores: puesto que los menos competitivos ganan más en eficiencia tras la apertura, estos aumentan la demanda de factores rivales desviando recursos hacia su sector en detrimento de los otros sectores.

Palabras clave: Comercio Internacional, Innovación, Modelos multisectoriales.

Códigos JEL: F13, F43, O3

a Antonio Navas Ruiz. Departamento de Fundamentos del Análisis Económico. Facultad de Ciencias Económicas y Empresariales. Campus de San Vicente del Raspeig. 03080. Alicante. España. Correo electrónico: antonionavas@merlin.fae.ua.es. El autor agradece la financiación recibida del Ministerio de Ciencia e Innovación bajo el programa ECO2008-01300.

Recibido: noviembre 2009 / Aceptado: mayo 2010 


\begin{abstract}
This paper explores the relationship between trade openness, competition and productivity growth, in a multi-sector R\&D endogenous growth model where we allow for sectorial differences in competition levels. A movement from autarky to free trade extends the size of the market but it increases competition within each market. The joint effect rises the output produced per firm, $R \& D$ employment and productivity growth. When we allow for sectorial differences, the less competitive sectors obtain larger gains in efficiency, increasing by more R\&D investment and productivity growth. Moreover, R\&D employment in the most competitive sectors may reduce due to a crowding-out effect from the less competitive sectors: The latter, increases the demand for rival inputs, attracting labor from $\mathrm{R} \& \mathrm{D}$ and production activities of the other sectors.
\end{abstract}

Keywords: International trade, Innovation, Multisectorial models.

JEL Classification: F13, F43, O3

\title{
1. INTRODUCCIÓN
}

Durante las últimas dos décadas hemos asistido a una explosión del comercio internacional. Dicha explosión ha afectado tanto a los países desarrollados, quienes dominan claramente los flujos del comercio mundial, como a los países en desarrollo cuya importancia relativa ha ido creciendo paulatinamente a partir de los años 90 (UNCTAD (2002)). ${ }^{1}$ Al mismo tiempo, hemos observado algunos intentos para crear acuerdos de integración regional, tales como la Unión Europea, MERCOSUR y NAFTA. Estos cambios han reavivado el interés que los economistas tienen sobre cuáles son los efectos sobre la productividad y el crecimiento de la apertura de una economía a los mercados internacionales.

1 Durante el período que va del año 1980 al año 2000 las exportaciones de los países en desarrollo crecieron a un ritmo mucho mayor que la media mundial y para el año 2002, estas constituían casi un tercio del volumen de comercio mundial (UNCTAD, World Investment Report 2002). 
La mayoría de los estudios tanto a nivel teórico como a nivel empírico se han centrado en analizar las consecuencias que la liberalización del comercio tiene en la productividad, tanto a nivel de empresa como a nivel de industria. En los últimos años, han adquirido especial relevancia los trabajos de Marc Melitz (2003) y Bernard, Jensen y cols. (2003), que centran la atención en los efectos sobre la productividad media de una industria, considerando un modelo con empresas heterogéneas. Motivado por la reciente evidencia empírica sobre la existencia de diferencias importantes entre empresas tanto en el volumen de exportaciones como en el estatus (exportador) dentro de una misma industria, Melitz (2003) construye un modelo donde las empresas difieren en productividad, y existen costes fijos inherentes tanto a la producción como a la actividad exportadora. Este resalta el impacto positivo que la liberalización al comercio supone para la productividad media de una industria a través de lo que el llama el efecto selección: Dado que las empresas tienen que invertir en costes fijos para poder exportar, sólo las empresas lo suficientemente productivas pueden exportar. Cuando la economía se abre al comercio internacional, las empresas domésticas menos productivas no pueden cubrir los costes fijos de producir en el mercado, ya que sufren mayor competencia y no pueden exportar, y deben salir del mercado. Esto hace subir la productividad media de la industria al quedarse únicamente, las empresas más eficientes. Este trabajo, sin embargo no considera cuáles son los efectos sobre la productividad a nivel de planta. ${ }^{2}$

En este sentido, otros trabajos como los de Navas y Sala (2007), De Long y cols. (2009), y Atkeson y Burstein (2010) han explorado el papel que el efecto selección juega en la decisión de las empresas a adoptar tecnologías más eficientes y por tanto a mejorar la productividad a nivel de planta. Los primeros dos artículos derivan que la apertura a los mercados internacionales genera una reasignación de recursos de las empresas menos eficientes a las empresas más eficientes, lo cual incentiva a estas últimas a adoptar nuevas tecnologías que suponen un aumento en la productividad. El último de ellos, trata de ver qué impacto tiene la apertura al comercio en el nivel de productividad

${ }^{2}$ Bernard, Jensen y cols. (2003) destacan también el efecto selección aunque desde un enfoque distinto: En su modelo tenemos una economía donde una variedad de empresas producen un mismo producto en un continuo de variedades. En equilibrio únicamente sobrevive la empresa más eficiente. Cuando la economía se abre al comercio, es la empresa más eficiente en el mundo la única que sobrevivirá, y todos los países aumentan su productividad media al ser servidos únicamente por las empresas más eficientes del mercado mundial. Aunque teóricamente distintas, sus conclusiones son en general similares a las expuestas en Melitz (2003). 
agregada. Estos encuentran que en general el efecto es nulo, o pequeño, para situaciones donde la elasticidad-empleo en el sector de innovación es alta.

Desde el punto de vista empírico varios son los trabajos que destacan el papel positivo que la liberalización del comercio ha tenido sobre la productividad y la innovación tanto a nivel de empresa como a nivel de industria. Bloom, Draca y Van Reenen (2009) encuentran que la penetración de importaciones chinas en la Unión Europea, si bien ha provocado la salida de varias empresas, también ha promovido la innovación en las empresas que han sobrevivido. ${ }^{3}$ Lileeva y Trefler (2008) encuentran que la reducción de aranceles en el tratado de libre comercio firmado por Canadá y Estados Unidos, en general incrementó la productividad a nivel de empresa, aunque el efecto fue mayor para empresas con bajo nivel de productividad, siendo casi nulo para empresas con un nivel de productividad alta. Bustos (2008) también encuentra que la apertura al comercio aumentó la proporción de empresas que adoptaron tecnologías más eficientes en Argentina. Otros artículos se centran más en el impacto que la liberalización tuvo sobre la productividad a nivel de industria (Pavcnick (2002), Topalova (2004)). ${ }^{4}$

Sorprendentemente, pocos trabajos han explorado cúal es el papel que la liberalización del comercio juega sobre la productividad a nivel de empresa, y a nivel de industria en modelos de equilibrio general que consideren diferencias sectoriales. Sin embargo, los procesos de liberalización comercial entrañan reducciones arancelarias y eliminación de barreras comerciales que suelen ser distintas entre sectores. A su vez, estos sectores difieren entre sí, en varias dimensiones. (competitividad, tamaño de mercado, desarrollo tecnológico, etc...). Parece sensato pues, estudiar los efectos de una liberalización comercial que conlleve reducciones asimétricas en barreras comerciales en un entorno caracterizado por la existencia de diferencias intersectoriales.

En este artículo nos centramos en una dimensión particular en la que los sectores se diferencian: el grado de competencia. Estudiamos cuales son las consecuencias que esto tiene en el efecto que la apertura a los mercados internacionales provoca en los niveles de productividad y su crecimiento. La razón fundamental descansa en el hecho de que el nivel de competencia de un sector es un determinante fundamental en la decisión de innovación por parte de las empresas. ${ }^{5}$

${ }^{3}$ La actividad innovadora se ha medido utilizando diversas fuentes, el número de patentes, la intensidad de I+D y la adopción de nuevas tecnologías en el sector de telecomunicaciones.

${ }^{4}$ Véase Tiebout (2003) para una revisión de la literatura empírica.

5 La primera generación de modelos de crecimiento endógeno predecían un efecto negativo del aumento de la competencia sobre los beneficios de innovar debido al espíritu schumpeteriano 
Para ello utilizamos una extensión del modelo desarrollado previamente en el artículo de Licandro y Navas (2010) que se centra fundamentalmente en analizar cuáles son los efectos que la liberalización al comercio tiene sobre la productividad y el crecimiento de una economía a través de un aumento de la competencia. Para ello, estos autores desarrollan un modelo de equilibrio general con dos sectores, uno de ellos con competencia oligopolística: un bien diferenciado compuesto de un continúo de variedades en donde en cada variedad existe un número determinado de empresas que ofrecen un producto homogéneo compitiendo á la Cournot.

Cuando la economía se abre al comercio internacional se producen dos cambios significativos a nivel de empresa: Por un lado cada empresa tiene acceso a un nuevo mercado y por tanto esto aumenta su demanda y su volumen de producción. Por otro lado la empresa sufre de una competencia mayor en cada uno de sus mercados. ${ }^{6} \mathrm{El}$ aumento de competencia hará que, a pesar de que el volumen de producción de cada empresa en cada uno de los dos mercados disminuya, el volumen total de producción de cada empresa aumente, aumentando los beneficios a innovar y por tanto aumentando el crecimiento de la productividad de cada empresa. El modelo reproduce fielmente la evidencia empírica que se ha encontrado sobre los efectos de la liberalización comercial en una industria particular: reducción de los márgenes por empresa debido al incremento de la competencia y aumento de los recursos destinados a innovación.

Por otro lado, descubriremos que la apertura de la economía a los mercados internacionales generará un efecto positivo mayor, cuanto menor es el nivel de competencia inicial del sector. Esto se debe al hecho de que el efecto competencia, es no lineal: Cuando hay pocas empresas en el mercado, un aumento proporcional del número de empresas, debido a la apertura al comercio, supone un aumento muy fuerte en la competencia, lo cual incentiva a las empresas a dedicar más recursos a innovar. A medida que el nivel de competencia es más alto el aumento proporcional del número de empresas supone un aumento menor en la competencia y por tanto en los esfuerzos a innovar por empresa. En el límite, cuando los sectores se aproximan a competencia perfecta,

que se escondía tras estos modelos (Romer (1990), Aghion y Howitt (1992)). Otra segunda generación de modelos consideraba también dentro de los beneficios de innovar, las consecuencias de no innovar si los demás innovan, efecto que denominaron «huir de la competencia» ( «escape from competition»): Aghion y cols. (2005).

${ }^{6}$ Suponemos que el conjunto de variedades que cada país produce es exactamente el mismo. Cuando la economía se abre al comercio, el número de productores por sector al que cada empresa se enfrenta en cada mercado se duplica. 
abrirse al comercio internacional no supondrá ninguna ganancia en términos de competencia y por tanto en términos de productividad.

El artículo descubre también otros efectos interesantes cuando consideramos diferencias sectoriales. La apertura al comercio de un único sector, genera descensos tanto en el volumen de producción como en los esfuerzos a innovar en el resto de los sectores situados al mismo nivel. Abrirse a los mercados extranjeros hace que un sector se vuelva más eficiente y productivo, provocando un efecto crowding-out sobre los factores de producción rivales con los que compite con otros sectores. Este segundo efecto tiene consecuencias interesantes para los experimentos que hemos llevado a cabo: si existen enormes diferencias de competencia a nivel sectorial, una apertura global a los mercados internacionales puede generar el efecto contrario al esperado en los sectores más competitivos. Esto es así porque aquellos sectores que son menos competitivos, se volverán más eficientes, atrayendo una gran parte de los recursos disponibles de la economía en detrimento de los otros sectores.

El artículo advierte de la cautela con la que se deberían hacer los ejercicios empíricos que tratan de establecer conclusiones sobre los efectos de la apertura al comercio sobre la productividad a nivel de industria y a nivel de empresa. Para la empresa media ${ }^{7}$ la apertura al comercio podría tener efectos negativos en los esfuerzos a innovar a nivel de planta, si en la economía se ha producido una apertura global y la economía cuenta con diferencias sectoriales importantes en niveles de competencia. El mismo resultado se podría obtener si la apertura se ha llevado a cabo de forma asimétrica, es decir, la liberalización no se ha producido en la misma magnitud en todos los sectores.

Dentro de la literatura teórica nuestro artículo se aproxima al trabajo llevado a cabo por Ederington y McCalman (2007). Este artículo examina cuales son los efectos de una reducción unilateral de barreras arancelarias en la adopción de tecnologías más eficientes. El modelo advierte que la reducción de tarifas arancelarias puede tener efectos distintos en la productividad tanto a nivel de empresa como a nivel de industria, atendiendo a distintas características tanto de planta como de industria. El modelo no obstante difiere con el que se encuentra en el presente artículo en bastantes aspectos. Por un lado el modelo no incluye de manera explícita diferencias en competencia, al no

${ }^{7}$ En esta versión del modelo todas las empresas son iguales. Licandro e Impulliti (2010) han extendido el modelo original de Licandro y Navas (2010) a un entorno de empresas heterogéneas para ver como se interrelacionan el efecto selección y el efecto competencia que comentamos aquí. Aunque el objetivo de esta simplificación haya sido ilustrar de manera adecuada los mecanismos del modelo extenderé en otro artículo el modelo a un entorno de empresas heterogéneas. 
incluir interacción estratégica entre empresas. A su vez, el enfoque es de equilibrio parcial y por tanto no considera efectos del tipo crowding-out de los que hemos discutido antes. Por otra parte, el autor lleva a cabo ejercicios totalmente distintos en el ámbito de la política comercial.

Por último cabe decir, que la apertura al comercio internacional se produce entre economías idénticas pero con diferencias sectoriales en niveles de competencia. En este artículo estamos mirando únicamente a diferencias de carácter sectorial, pero no estamos atendiendo a diferencias en ventajas comparativas entre países. Este ejercicio debe entenderse en un marco de apertura al comercio (acuerdo regional), entre países con niveles similares de tecnología, competencia, etc..El modelo no analiza casos de comercio norte-sur. No obstante el $70 \%$ de los flujos de comercio mundial en el año 2002 todavía eran del tipo norte-norte (entre países desarrollados) y en la última década hemos visto una tendencia creciente en el papel del comercio sur-sur (entre países en desarrollo). ${ }^{8}$

El artículo consta de varias secciones. En la primera sección exponemos el modelo en autarquía, explicando los mecanismos básicos del artículo original de Licandro y Navas (2010) así como los nuevos elementos. En la segunda sección consideramos un escenario en la que dos economías idénticas se abren al comercio en todos sus sectores y comentamos también los principales mecanismos del modelo. Puesto que no existe una solución analítica del modelo en la sección tercera realizamos un ejercicio de simulación donde mostramos los principales resultados del modelo. En la sección cuarta concluimos.

\section{EL MODELO}

\subsection{Los consumidores}

Consideremos una economía habitada por un continuo de hogares de medida $L$ en la que existen dos tipos de bienes finales: El bien $Y_{t}$ que podríamos denotar como bien tradicional, y el bien $X_{t}$ que podríamos llamar bien industrial. Los hogares derivan utilidad del consumo de ambos bienes y su función de utilidad instantánea viene dada por:

$$
U\left(X_{t}, Y_{t}\right)=\beta \ln X_{t}+(1-\beta) \ln Y_{t}
$$

${ }^{8}$ UNCTAD, World Investment Report (2002). 
donde el parámetro $\beta$ nos indica el peso del bien $X_{t}$ en la función de utilidad. La producción del bien $Y_{t}$ se lleva a cabo mediante una tecnología líneal en trabajo y por tanto su función de producción viene dada por:

$$
Y_{t}=L_{t}^{y}
$$

Asumimos que existe competencia perfecta en la producción del bien $Y_{t}$ y que el precio del bien Asumimos que existe competencia perfecta en la producción del bien $Y_{t}$ y que el precio del bien $Y_{t}$ es normalizado a uno. Esto nos llevará a un salario de equilibrio de $w_{t}$. El bien $X_{t}$ se trata de un bien diferenciado y suponemos que existe un continuo de variedades de medida uno. Consideramos que las distintas variedades se agregan mediante la siguiente forma funcional: es normalizado a uno.

$$
X_{t}=\int_{0}^{1} \ln x_{i t} d t
$$

donde $x_{i t}$ denota la cantidad consumida de la variedad $i$ en el momento $t$. Al suponer dicha forma funcional, estamos asumiendo que los individuos tienen preferencias logarítmicas sobre las distintas variedades. Éste supuesto, por otra parte, es necesario, para simplificar el modelo en el ámbito de estudio que nos hemos marcado: sabemos que en presencia de diferencias intersectoriales en las tasas de crecimiento de la productividad agregada, esta función de utilidad nos garantiza la existencia de una senda de crecimiento equilibrado en la que la asignación intersectorial de trabajo permanece constante a lo largo del tiempo (Ngai and Pissarides [2006]). Podríamos suponer una forma más genérica para la función de utilidad, del tipo CES como en el artículo original de Licandro y Navas (2010), pero la asignación intertemporal de trabajo no sería constante en la senda de crecimiento equilibrado, lo cual complicaría bastante el ejercicio, sin tampoco aportar demasiado al estudio. La noción de cambio estructural está fuera del alcance del presente estudio. ${ }^{9}$

${ }^{9}$ Es importante tener en cuenta, no obstante que el supuesto de preferencias Cobb-Douglas a nivel más agregado probablemente sea consistente con la evidencia empírica. Los individuos tienden a dedicar una proporción constante de su renta a consumir determinados grupos de bienes, ropa, calzado, comida, electrodomésticos, etc. Mientras que suele haber sustitución entre distintos bienes a niveles más desagregados (entre distintas tipos o marcas de televisores, o entre distintas variedades de una prenda de vestir en concreto, etc.). 
En cada una de estas variedades hay $n_{i}$ empresas que ofertan el producto. Estas $n_{i}$ empresas utilizan la siguiente tecnología lineal:

$$
q_{i j t}=z_{i j t} l^{x}{ }_{i j t}
$$

dónde $q_{i j t}$ denota la cantidad producida de la variedad $i$ por la empresa $j$ en el momento $t$. y $z_{i j t}$ es una medida del conocimiento específico de la empresa. Este elemento engloba muchas formas de capital intangible: capital organizativo, know-how, etc.., que constituyen la ventaja competitiva específica de la empresa. Llamamos a la fuerza laboral dedicada a la producción por empresa $l_{i j t}^{x}$. Las empresas compiten á la Cournot en cantidades.

Las empresas llevan a cabo actividades de investigación y desarrollo (I+D) con el fin de aumentar el conocimiento específico de la empresa $z_{i j t}$. En concreto la empresa contrata trabajadores para su departamento de investigación, los cuales producen nuevo conocimiento de acuerdo con la siguiente función de producción:

$$
\dot{z}_{i j t}=\left(l_{i j t}^{z}\right)^{\gamma} z_{i j t} \quad 0<\gamma<1
$$

donde el aumento en el stock de conocimiento es fruto del conocimiento acumulado de la empresa (spillovers tecnológicos internos a la empresa) y de la fuerza laboral dedicada a investigación y desarrollo. El parámetro $\gamma$ mide la contribución que el factor trabajo tiene en la creación de nuevo conocimiento $(0<\gamma \leq 1)$.

Para financiar las inversiones en (I+D) las empresas emiten nuevas acciones $\left(A_{t}\right)$. Suponemos que inicialmente todos los individuos poseen el mismo número de acciones de cada empresa que existe en el mercado. Los individuos al adquirir estas acciones contribuyen a financiar los proyectos de inversión en (I+D) de las empresas obteniendo a cambio unos retornos $r_{t}$.

Los individuos resolverán el siguiente problema estándar de maximización intertemporal:

$$
\begin{aligned}
& \operatorname{Max} \int_{0}^{\infty} e^{-\rho t}\left(\beta \ln X_{t}+(1-\beta) \ln Y_{t}\right) d t, \quad \rho>0, \\
& \text { s.a. } \quad X_{t}=\int_{0}^{1} \ln x_{i t} d t \\
& \dot{A}_{t}=w_{t}+r_{t} A_{t}-\int_{0}^{1} p_{i t} x_{i t} d i-C_{t}^{y}, \quad A_{0} \text { dado }
\end{aligned}
$$




\subsection{Las empresas}

Las empresas deben elegir para cada período de tiempo. la cantidad que ofrecen al mercado $q_{i j T}$ y los recursos que dedican a investigación $l_{i j t}^{z}$ tomando como dadas las estrategias de las otras empresas. Este juego se enmarca dentro de un subconjunto particular de juegos dinámicos denominados juegos diferenciales. Para estos juegos se han propuesto dos definiciones básicas de equilibrio de Nash: El equilibrio de Nash llamado open loop y el llamado closed loop. En un equilibrio de Nash del tipo open loop las empresas en el momento $t=0$, deciden el plan de estrategias óptimo para cada período y se ciñen a este plan durante el período de vida del juego. En un equilibrio llamado closed loop las empresas deciden en cada momento del tiempo el plan de estrategias óptimo para cada período. En cierto modo, el equilibrio de Nash del tipo open loop es un equillibrio básicamente estático ya que la empresa en el momento $t=0$, decide su plan óptimo para todo el período de vida de la empresa y por tanto no observa la estrategia de las otras empresas.

Los desarrollos de esta rama de la teoría de juegos han intentado buscar bajo que condiciones podríamos asegurar que el equilibrio de Nash del tipo open loop es un equilibrio de Nash del tipo closed loop y por tanto es perfecto en subjuegos o consistente en el tiempo. Básicamente esto se ha hecho por la dificultad que conlleva buscar equilibrios de Nash del tipo closed loop en estos juegos. Nosotros podemos asegurar que en nuestro caso los equilibrios de Nash del tipo open loop son closed loop y por tanto perfecto en subjuegos. ${ }^{10}$ Por consiguiente nos centraremos en este tipo de equilibrios. Además nos centraremos en equilibrios simétricos suponiendo que $z_{i j 0}=z_{i k 0}$ $\neg j$, $k$. como viene siendo estándar en esta literatura (Peretto [2003], Cellini and Lambertini [2005], etc.).

Definamos $a_{i j T}=\left[q_{i j T}, l_{i j T}^{z}\right], \neg T \geq t$, como el vector de estrategias de la empresa j en el sector $\mathrm{i}$, donde $\left[q_{i j T}, l^{z}{ }_{i j T}\right]$ son secuencias de cantidades y de trabajadores en el sector de I+D elegidos por la empresa j para cada momento del tiempo y definamos $\Omega_{i}$ como el conjunto de posibles estrategias de la empresa $j$. Denotemos también con $V_{i j T}$ el valor

${ }^{10}$ La prueba se reduce a mostrar que las condiciones de primer orden asociadas al hamiltoniano de cada empresa no dependen directamente de la variable estado de los rivales. Como referencias técnicas se pueden utilizar Müller (1982), Fehrstmann (1987), Reinganum (1982). Una aplicación de estos resultados la podemos encontrar en Cellini and Lambertini (2005). 
de la empresa j cuando las $n$ empresas de cada mercado $n \geq 2$, juegan las estrategias $A_{i n T}=\left[a_{i 1 T}, a_{i 2 T}, \ldots, a_{i n T}\right]$. Entonces tenemos que:

$$
V_{i n T}\left[A_{i n T}\right] \geq V_{i j T}\left[A_{i n T} T^{\prime}\right] \geq 0
$$

donde $A_{i n T^{\prime}}=\left[a_{j T^{\prime}}, a_{-j T}^{*}\right], \neg a_{j T^{\prime}} \alpha \Omega_{j}$.

\subsection{Solución del modelo}

Para empezar a resolver el modelo comencemos resolviendo el problema del consumidor. Fijémonos primero en que la función de utilidad es perfectamente separable lo cual nos permite concentrarnos en cada una de las decisiones que atañen al consumidor de manera autónoma. La decisión intertemporal de asignación de recursos viene dada por la ecuación de Euler:

$$
\frac{\dot{E}}{E}=r_{t}-\rho
$$

donde $E$ denota la parte de recursos que se dedica al gasto en bienes por hogar. Este gasto en bienes se distribuirá entre cada uno de los dos bienes $X_{t}, Y$ en proporciones fijas, ya que tenemos preferencias Cobb-Douglas, y por tanto:

$$
\begin{gathered}
E_{t}^{x}=\beta E t \\
E_{t}^{Y}=(1-\beta) E_{t}
\end{gathered}
$$

Por último los recursos dedicados al consumo del bien $X_{-}\{t\}$ se distribuirán uniformemente entre cada una de las distintas variedades a partes iguales, en concreto:

$$
p_{i t} x_{i t}=p_{k t} x_{k t} \neg i, k
$$

y la función de demanda agregada para cada una de las variedades viene dada por:

$$
p_{i t}=\frac{L E_{t}^{x}}{x_{i t}} .
$$


La empresa resuelve el siguiente problema de optimización:

$$
V_{i j s}=\max \int_{0}^{\infty} R_{s, t}\left(\left(p_{i t}-z_{i j t}{ }^{-1}\right) q_{i j t}-l_{i j t}^{z}\right) d t,
$$

Sujeto a las siguientes restricciones,

$$
\begin{gathered}
p_{i t}=\frac{L E_{t}^{x}}{x_{i t}} . \\
x_{i t}=\sum_{i=1}^{n_{j}} q_{i j t} \\
\dot{z}_{i j}=\left(l_{i j t}^{z}\right)^{\gamma} z_{i j t} \quad 0<\gamma<1 \\
z_{i 0}>0
\end{gathered}
$$

donde Rs, $\mathrm{t}=\mathrm{e}^{-\int_{\mathrm{s}}^{\mathrm{t}} \mathrm{t} d z}$ es el factor de descuento estándar de la empresa. Las condiciones de primer orden asociadas al valor corriente del hamiltoniano, vienen dadas por:

$$
\begin{gathered}
\frac{L E_{t}^{x}}{x_{i t}}-\frac{L E_{t}^{x}}{x^{2}{ }_{i t}} q_{i j t}-z^{-1}{ }_{i j t}=0 \\
1-\gamma v_{i j t}\left(l_{i j t}^{z}\right)^{\gamma-1} z_{i j t}=0 \\
z_{i t}^{-2} q_{i t}+\left(l_{i t}^{z}\right)^{\gamma} v_{i t}=-\dot{v}_{i}+r_{t} v
\end{gathered}
$$

considerando el equilibrio simétrico estas vienen dadas por:

$$
q_{i t}=\theta_{i} z_{i t} \frac{L}{n} E_{t}
$$




$$
\begin{gathered}
1=\gamma v_{i t}\left(l_{i t}^{z}\right)^{\gamma-1} z_{i t}, \\
\frac{z_{i t}{ }^{-2} q_{i t}}{v_{i t}}+\left(l_{i t}^{z}\right)^{\gamma}=\frac{-\dot{v}_{i}}{v_{i}}+r_{t}
\end{gathered}
$$

La condición es la asociada a la variable estado $z_{i t}$ y por tanto nos informa sobre la decisión óptima de invertir en conocimiento. En la parte izquierda observamos los beneficios que la empresa obtiene al aumentar marginalmente su conocimiento, revelando la importancia que tiene la cantidad producida. Al ser una innovación cuyo objetivo es el incremento de la productividad o la reducción en los costes marginales de producción, los beneficios de innovar son mayores cuanto mayor es el número de unidades que la empresa produce y por tanto mayor es el ahorro en recursos de producir tal cantidad. Este ingrediente como expusimos en Licandro y Navas (2010) es esencial en los resultados posteriores. El segundo término de la izquierda recoge los beneficios que nos da el hecho de que existan spillovers tecnológicos: Al aumentar marginalmente mi conocimiento actual reduzco el coste de producir conocimiento futuro. Del lado de la derecha, se encuentran los costes de aumentar ese conocimiento que vienen dados por el coste de oportunidad de mercado $r_{t}$, así cómo la variación en el valor de la innovación.

Dado que la cantidad a producir es un ingrediente esencial en los resultados posteriores, debemos comentar como se ve determinada en el modelo de Cournot. La condición es la encargada de darnos esa información. El primer término de esa condición nos da los beneficios que la empresa obtiene al aumentar la cantidad producida tomando como dadas las cantidades producidas de las otras empresas. En concreto, el primer término de la condición, nos da el beneficio de aumentar la cantidad a producir si el precio del bien no cambiase con el aumento de tal cantidad, mientras que el segundo nos da las pérdidas que tendría la empresa al aumentar la cantidad debido a la caida del precio del bien. Fijémonos que, a medida que $x_{i t}$ es más grande, el segundo efecto es menor, y por tanto la empresa tiene incentivos a ofrecer una cantidad mayor en el mercado.

Puesto que la liberalización al comercio supone por un lado mayor competencia y por otro un aumento del tamaño de mercado es interesante ver cuales son los efectos de un aumento del número de competidores en la cantidad a producir de cada empresa.

El aumento del número de competidores tiene en un principio dos efectos en la cantidad a producir por empresa: Por un lado un aumento del número de empresas reduce la cuota de mercado de cada empresa reduciendo la cantidad que produce. A este 
efecto se le conoce en la literatura como market share effect, o efecto de la cuota de mercado (Vives [2007]); por otro lado, el aumento del número de empresas reduce el márgen que las empresas cargan a su producto aumentando la cantidad a producir de cada empresa. Este segundo efecto, conocido en la literatura como efecto competencia o efecto interacción estratégica, proviene del hecho de que un aumento en el número de empresas, aumenta la elasticidad percibida de demanda, aumentando los incentivos de la empresa a ofrecer más cantidad en el mercado. ${ }^{11}$

Finalmente, imponemos las condiciones de vaciado de mercado para resolver el equilibrio. De aquí en adelante introduciremos el análisis de diferencias intersectoriales a nivel de competencia, que es precisamente el objeto de nuestro estudio. Para simplificar este análisis conviene considerar que las distintas variedades del bien $X_{t}$ se agrupan en dos tipos de subsectores: los subsectores 1,2. Estos subsectores podrán ser diferentes entre sí en términos de competencia, pero serán iguales entre variedades de un mismo subsector. Vamos a considerar sin perder la generalidad que cada subsector tiene la misma masa de variedades que el otro subsector.

Entonces la condición de vaciado de mercado de trabajo de nuestra economía viene dada por:

$$
L_{1 t}^{x}+L_{1 t}^{z}+L_{2 t}^{x}+L_{2 t}^{z}+L_{t}^{y}=L
$$

donde denotamos $L_{i t}^{x}, L_{i t}^{z}$ respectivamente como la asignación de trabajo total en cada una de las actividades en cada uno de los subsectores. La condición de vacíado de mercado de stocks viene dada por:

$$
L A_{t}=\frac{n_{1} V_{1 t}+n_{2} V_{2 t}}{2}
$$

11 Para estas preferencias la elasticidad precio de demanda es uno, mientras que la elasticidad percibida de demanda es n. Las empresas en el modelo de Cournot tienen un tradeoff entre ofrecer más cantidad en el mercado a costa de bajar el precio de equilibrio y por tanto la ganancia por unidad. Este tradeoff está gobernado por dicha elasticidad. A mayor elasticidad menor es el efecto que un aumento de la cantidad que cada empresa oferta en el mercado tiene sobre el precio de equilibrio y por tanto mayores son los beneficios de ofrecer más en el mercado. Una referencia más técnica la puede encontrar en el apéndice. 
donde la cantidad de stocks que poseen los consumidores igualan al valor de todas las empresas de la economía, y por último nos queda la condición de vacíado de mercado del bien final $Y_{t}$ :

$$
L E_{t}^{Y}=L_{t}^{y}
$$

Senda de crecimiento equilibrado en autarquía

Una senda de crecimiento equilibrado en esta economía es una situación donde las variables $L_{i t}^{x}, L_{i t}^{z}, l_{i j t}^{x}, l_{i j t}^{z}, r_{t}, E_{t}, E_{t}^{y}, L_{t}^{Y}, Y$ son constantes a lo largo del tiempo y donde las variables $q_{t}$ crecen a una tasa constante.

Proposición: En esta economía tenemos una senda de crecimiento equilibrado que además es única.

Prueba: Démonos cuenta que en una senda de crecimiento equilibrado las variables $L_{i t}^{x}, L_{i t}^{z}, l_{i j t}^{x}, l_{i j t}^{z}, L^{Y}$, deben ser constantes porque están acotadas superiormente.

Consideremos la variable $E^{y}{ }_{t}$. Puesto que $L^{Y}{ }_{t}$ es constante es constante y por consiguiente en la senda de crecimiento equilibrado $r_{t}=\rho$.

Combinando (0.2), (0.3) y (0.6) bajo $i_{z}=0$ obtenemos:

$$
\gamma\left(l_{j}^{z}\right)^{\gamma-1} l_{j}^{x}=\rho . \quad j=1,2
$$

Esta condición nos dice que los consumidores son indiferentes entre invertir en activos del subsector 1 , y en activos del subsector 2, En estado estacionario la tasa de retorno de ambos debe ser igual al factor de descuento como hemos obtenido anteriormente Esto implica que:

$$
\frac{l_{1}^{z}}{l_{2}^{z}}=\left(\frac{l^{x}{ }_{1}}{l_{2}^{x}}\right)^{\frac{1}{1-\gamma}}
$$

donde de nuevo se revela la complementariedad entre la demanda de trabajo en la producción y la demanda de trabajo dedicado a I+D. Aquellas empresas que son más grandes, son también las que dedican más esfuerzos a innovar.

En el equilibrio simétrico tenemos que $x_{i t}=n_{i} q_{i t}$. Combinando esto con y el hecho de que: 


$$
P_{i t}=\frac{1}{\theta_{i}} z_{i}^{-1}, \quad i=1,2
$$

tenemos que

$$
\frac{n_{1}}{\theta_{1}} l_{1 t}^{x}=\frac{n_{2}}{\theta_{2}} l_{2 t}^{x}
$$

y entonces

$$
\begin{gathered}
\frac{l^{x}{ }_{2 t}}{l^{x}{ }_{1 t}}=\frac{\theta_{2}}{\theta_{1}} \frac{n_{1}}{n_{2}} \\
l^{x}=\frac{\rho}{\gamma}\left(l_{j}^{z}\right)^{1-\gamma}, \quad j=1,2
\end{gathered}
$$

Démonos cuenta de que la demanda de trabajo dedicado a la producción, es mayor, en sectores caracterizados por menos competencia. Este efecto es estándar en el modelo de Cournot. Cuando aumenta el número de competidores el efecto de la cuota de mercado siempre domina al efecto de competencia y por tanto más competencia implica menor producción por empresa, aunque el volumen total de producción de la industria crezca con el número de empresas.

Para ser capaz de conseguir una expresión para la asignación de equilibrio de trabajadores en cada actividad y en cada sector necesitamos combinar un poco más las condiciones de primer orden. Démonos cuenta que:

$$
l_{j}^{x}=\frac{\rho}{\gamma}\left(l_{j}^{z}\right)^{1-\gamma}, \quad j=1,2
$$

Con estas cuatro ecuaciones ya podemos expresar la condición de vaciado de mercado en función de la variable $l_{2}$. En concreto tenemos que: 


$$
\begin{gathered}
l_{2}^{x}=\frac{\rho}{\gamma}\left(l_{2}^{z}\right)^{1-\gamma}, \quad j=1,2 \\
\frac{l_{2 t}^{x}}{l_{1 t}^{x}}=\left(\frac{\theta_{2}}{\theta_{1}} \frac{n_{1}}{n_{2}}\right) \frac{\rho}{\gamma}\left(l_{2}^{z}\right)^{1-\gamma} \\
\frac{l_{2 t}^{z}}{l_{1 t}^{z}}=\left(\frac{\theta_{2}}{\theta_{1}} \frac{n_{1}}{n_{2}}\right)^{\frac{1}{1-\gamma}}
\end{gathered}
$$

y obteniendo $L^{y}$ en función de $l_{2}^{z}$ tenemos que:

$$
L^{y}=\left(\frac{1-\beta}{\beta}\right) E^{x}=\left(\frac{1-\beta}{\beta}\right)\left(\frac{n_{1} p_{1} q_{1}+n_{2} p_{2} q_{2}}{2}\right)=\left(\frac{1-\beta}{\beta}\right) \frac{n_{2}}{\theta_{2}} \frac{\rho}{\gamma}\left(l_{2}^{z}\right)^{1-\gamma}
$$

Para llegar a la condición de vaciado de mercado de trabajo final démonos cuenta que:

$$
\begin{aligned}
& L_{1 t}^{s}=\int_{0}^{1 / 2} n_{1} l_{1}^{s}=\frac{n_{1}}{2} l_{1}^{s}, \quad s=x, z \\
& L_{2 t}^{s}=\int_{0}^{1 / 2} n_{2} l_{2}^{s}=\frac{n_{2}}{2} l_{2}^{s}, \quad s=x, z
\end{aligned}
$$

y combinando las ecuaciones anteriores llegamos a la condición de vaciado de mercado expresada en términos de $l_{2}^{2}$

$$
\left(\frac{2(1-\beta)+\beta\left(\theta_{1}+\theta_{2}\right)}{2 \beta \theta_{2}}\right) \frac{\rho}{\gamma}\left(l_{2}^{z}\right)^{1-\gamma}+\left(\left(\frac{\theta_{1}}{\theta_{2}}\right)^{(1 /(1-\gamma))}\left(\frac{n_{2}}{n_{1}}\right)^{(\gamma /(1-\gamma))}+1\right) l_{2}^{z}=l
$$


donde $l=\frac{L}{n_{2}}$.

Démonos cuenta que $\mathrm{f}\left(l_{2}^{2}\right)$ es monótona creciente y satisface las siguientes condiciones $\lim _{k_{2} \perp 0} f\left(l_{2}\right)=0$ y que $\lim _{k_{2} \perp l} f\left(l_{2}^{z}\right)>l$, satisfaciendo las condiciones del teorema del valor medio y por tanto demostrando la existencia y unicidad de la senda de crecimiento equilibrado.

Démonos cuenta que si $\beta=(1 / 2)$, y $\theta_{1}=\theta_{1}$ (i.e, $n_{1}=n_{2}$ ), es decir, los sectores son iguales y entran en la función de utilidad de la misma manera, tendremos que está condición es equivalente a la expuesta en Licandro y Navas (2010). No obstante, como en ese artículo se asumen preferencias del tipo CES, debemos suponer además que la elasticidad de sustitución entre variedades es igual a uno (Cobb-Douglas) para obtener la misma expresión.

Hemos visto anteriormente que un aumento en el número de empresas reducía los recursos destinados a investigación y desarrollo por empresa en este modelo. Sin embargo, al igual que en Licandro y Navas (2010) planteémonos un ejercicio distinto: supongamos que el grado de competencia aumenta $\theta_{i} \sin$ que cambie el número de empresas que producen en la economía. Este experimento, se aproxima mucho a una situación de apertura a los mercados internacionales sin barreras comerciales, ya que en ese contexto lo que aumenta son el número de competidores (de empresas que sirven un mercado) sin aumentar el número de empresas que producen en un mercado determinado. Examinando la ecuación anterior, vemos que el aumento de $\theta_{i}$ claramente aumenta los esfuerzos a innovar en el sector $i$.

Algo novedoso en este artículo, es que el aumento de $\theta_{i}$, conlleva claramente una disminución en los esfuerzos a innovar en el sector $j$. El aumento de $\theta_{i}$ aumenta la demanda de trabajo en el sector $i$ aumentando la competencia por el factor trabajo y reduciendo tanto la producción como los esfuerzos dedicados a la innovación en el sector j. El aumento de $\theta_{i}$ hace al sector i mucho más competitivo, haciendo bajar sus márgenes, aumentando la demanda del bien y desviando recursos del resto de sectores de la economía hacia la producción y la innovación en ese sector.

En la próxima sección consideraremos el caso de dos economías idénticas que se abren completamente al comercio internacional, considerando que no existen ningún tipo de barreras comerciales entre ellas. Veremos que en general, el aumento de la competencia que proviene de la apertura a los mercados internacionales, incrementará el volumen de producción total de la empresa, incrementando por consiguiente los incentivos a innovar. También veremos que aquellos sectores que comienzan en un 
principio con un menor nivel de competencia, serán los que al final aumentarán más los esfuerzos a la innovación.

\section{APERTURA AL COMERCIO EXTERIOR}

Consideremos ahora dos economías idénticas que en el momento $t=0$ se abren al comercio internacional en un mundo donde no existen costes de transporte. Las economías tienen el mismo tamaño de sectores y producen el mismo conjunto de variedades, pero existe un aumento en el número de productores que ofrecen una misma variedad.

El problema al que se enfrentan los consumidores es idéntico al de la sección anterior. Sin embargo el problema de la empresa difiere, pues ahora la empresa puede servir al mercado extranjero. Consideramos, como viene siendo estándar en la literatura, que la empresa trata a los dos mercados como mercados segmentados.

Las empresas resuelven el siguiente problema de maximización:

$$
V_{i j s}=\max \int_{s}^{\infty} R_{s, t}\left(\left(p_{i t}-z_{i j t}{ }^{-1}\right) q_{i j t}+\left(p^{*}{ }_{i t}-z_{i j t}{ }^{-1}\right) q^{*}{ }_{i j t}-l^{z}{ }_{i j t}\right) d t,
$$

Sujeto a las siguientes restricciones

$$
\begin{aligned}
& p_{i t}=\frac{L E^{x}{ }_{t}}{x_{i t}} . \\
& p_{i t}^{*}=\frac{L E^{x}}{x^{*}{ }_{i t}} . \\
& x_{i t}=\sum_{t-1}^{n_{j}} q_{i j t} \\
& x_{i t}^{*}=\sum_{t-1}^{n_{j}} q^{*}{ }_{i j t} \\
& \dot{z}_{i j}=\left(l^{z}{ }^{2}\right)^{\gamma} z_{i j t} \quad 0<\gamma<1 \\
& z_{i 0}>0
\end{aligned}
$$


donde ahora tenemos que las condiciones de primer orden del problema de las empresas en un equilibrio simétrico vienen dadas por:

$$
\begin{aligned}
& q_{i t}=\theta \propto_{i} z_{i t} \frac{L}{n} E_{t} \\
& q^{*}{ }_{i t}=\theta \propto_{i}^{*} z_{i t} \frac{L}{n} E_{t} \\
& 1=\gamma v_{i t}\left(l_{i t}^{z}\right)^{\gamma-1} z_{i t} \\
& \frac{z_{i t}^{-2}\left(q_{i t}+q_{i t}^{*}\right)}{v_{i t}}+\left(l_{i t}^{z}\right)^{\gamma}=\frac{-\dot{v}_{i}}{v_{i}}+\mathrm{r}_{\mathrm{t}}
\end{aligned}
$$

donde observamos en la cuarta condición, que la empresa considera la cantidad producida tanto en el mercado doméstico como en el mercado extranjero, a la hora de decidir cuantos recursos dedicar a innovación. Nótese también que, puesto que las dos economías son idénticas, $\theta \propto \propto_{i}=\theta_{\varkappa_{i}}^{*}$ y por consiguiente la empresa ofrecerá la misma cantidad en ambos mercados.

Teniendo en cuenta estas consideraciones, podemos definir una senda de crecimiento equilibrado para esta economía, donde fácilmente se puede verificar que la condición de vaciado de mercado en el mercado de trabajo es idéntica a la de autarquía pero donde en cada sector tendremos un $\theta$ ordiferente. En concreto:

$$
\theta \propto c_{i}=\frac{2 n_{i}-1}{2 n_{i}}
$$

y la condición de vaciado de mercado queda:

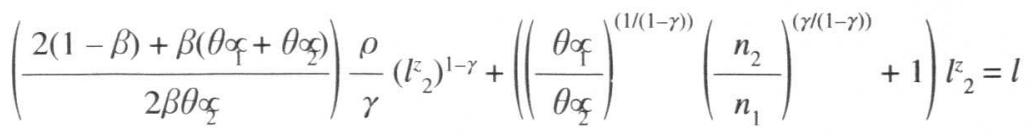

donde $\theta_{i}>\theta_{i}$ 
Fijémonos que no podemos sacar ninguna conclusión inicial sobre los efectos de la apertura al comercio puesto que el aumento de $\theta_{2}$ aumenta los esfuerzos a innovar en el sector 2, pero el aumento de $\theta_{1}$ provoca un efecto contrario.

El artículo de Licandro y Navas (2010) demuestra que la apertura a los mercados internacionales reduce la cantidad producida por empresa en cada uno de los mercados, pero aumenta la cantidad total producida por la empresa en su conjunto, cuando los sectores son completamente simétricos $\left(\theta \propto=\theta \alpha_{c}\right)$. Como los esfuerzos a innovar dependen de la cantidad global que produce la empresa, la apertura a los mercados internacionales aumenta los recursos destinados a la innovación. La intuición de este resultado descansa en el hecho de que cuando la economía se abre a los mercados internacionales, cada empresa tiene acceso a un mercado que es idéntico. Cada empresa sufre más competencia en cada uno de los dos mercados pero tiene acceso a ambos mercados. Esto último cancela el efecto de cuota de mercado del que habíamos hablado en la sección anterior, ya que la cuota de mercado de cada empresa en su conjunto no ha variado tras la apertura. Sin embargo el efecto competencia continúa ahí. El aumento de la competencia en cada uno de los mercados, a través de este efecto, aumenta el volumen de producción y los esfuerzos dedicados a innovar para cada empresa.

\subsection{Resultados numéricos}

Para responder a la pregunta relevante de este artículo hemos acudido a la simulación numérica. He considerado valores para los parámetros que sean consistentes con algunos hechos de la economía estadounidense. En ese sentido sigo cercanamente el artículo de Licandro and Impulliti (2010) que han realizado una extensión del modelo original de Licandro y Navas (2010) a un entorno con empresas heterogéneas.y han llevado este modelo a los datos.

En un principio debemos obtener valores para los parámetros $(\beta, \gamma, \rho, L)$. En estado estacionario el factor de descuento es igual al tipo de interés. En ese sentido tomamos el valor de 0.03 que es el que se ha utilizado comúnmente en la literatura de ciclos reales. Para el parámetro $\beta$ que mide el tamaño del sector no tradicional lo hemos elegido teniendo en cuenta que el peso de la agricultura en la economía estadounidense es del $9 \%$.

Por último nos queda por determinar el parámetro $\gamma$ y el parámetro L. Fijémonos que el parámetro $\gamma$ mide la elasticidad de innovación con respecto al empleo en investigación y desarrollo. Un aumento de un punto porcentual del número de trabajadores en investigación y desarrollo aumenta en $\sim$ puntos porcentuales el aumento en la produc- 
tividad de la empresa. En un principio sería complejo intentar calibrar este parámetro porque no es fácilmente observable en los datos. Por el momento escogeremos un valor arbitrario para este parámetro y haremos análisis de robustez. Puesto que el porcentaje de la población activa que se dedicaba al sector de investigación y desarrollo era de un $0.75 \%$ en Estados Unidos en 1990 (Jones, 2002), debemos considerar un valor bajo de este parámetro. Para el parámetro $L$ siguiendo Licandro e Impulliti (2010), he escogido $L=1.32$ que es la media de la fuerza laboral estadounidense para el período 1990-2000, reescalado para obtener los mejores resultados con el modelo.

La figura 1 muestra la variación porcentual en el nivel de empleo en investigación y desarrollo antes y después de la apertura al comercio en un entorno donde los sectores comienzan con el mismo nivel de competencia o mismo número de empresas. En todos los ejercicios que hemos considerado, siempre hemos incluido como restricción que los beneficios de las empresas sean mayores que cero tanto antes como después de la apertura al comercio. La figura corrobora lo ya comentado y mostrado en Licandro y Navas (2010): que la liberalización del comercio ha aumentado los esfuerzos dedicados a la innovación en ambos sectores.

Un resultado interesante, también comentado en Licandro y Navas (2010) es observar que el grado de competencia inicial influye en el aumento de los recursos a innovar. En concreto, cuando el nivel inicial de competencia es bajo, la apertura a los mercados internacionales, generará un aumento considerable de los esfuerzos dedicados a innovación. Esto se debe a que el efecto competencia se trata de un efecto no lineal: Es mucho más fuerte a medida que los sectores van siendo menos competitivos. En mercados caracterizados por altos niveles de competencia, la apertura de los mercados supondrá un incremento mínimo en la competencia, lo que se trasladará a un efecto mínimo en los recursos destinados a la innovación. Obsérvese que efectivamente, cuando el número de empresas es pequeño $(n=2,3)$ los efectos son importantes, pero a partir de 5 empresas, el aumento en el empleo en I+D es menor del $1 \%$. 
FIGURA 1.-Incremento porcentual en I+D en ambos sectores

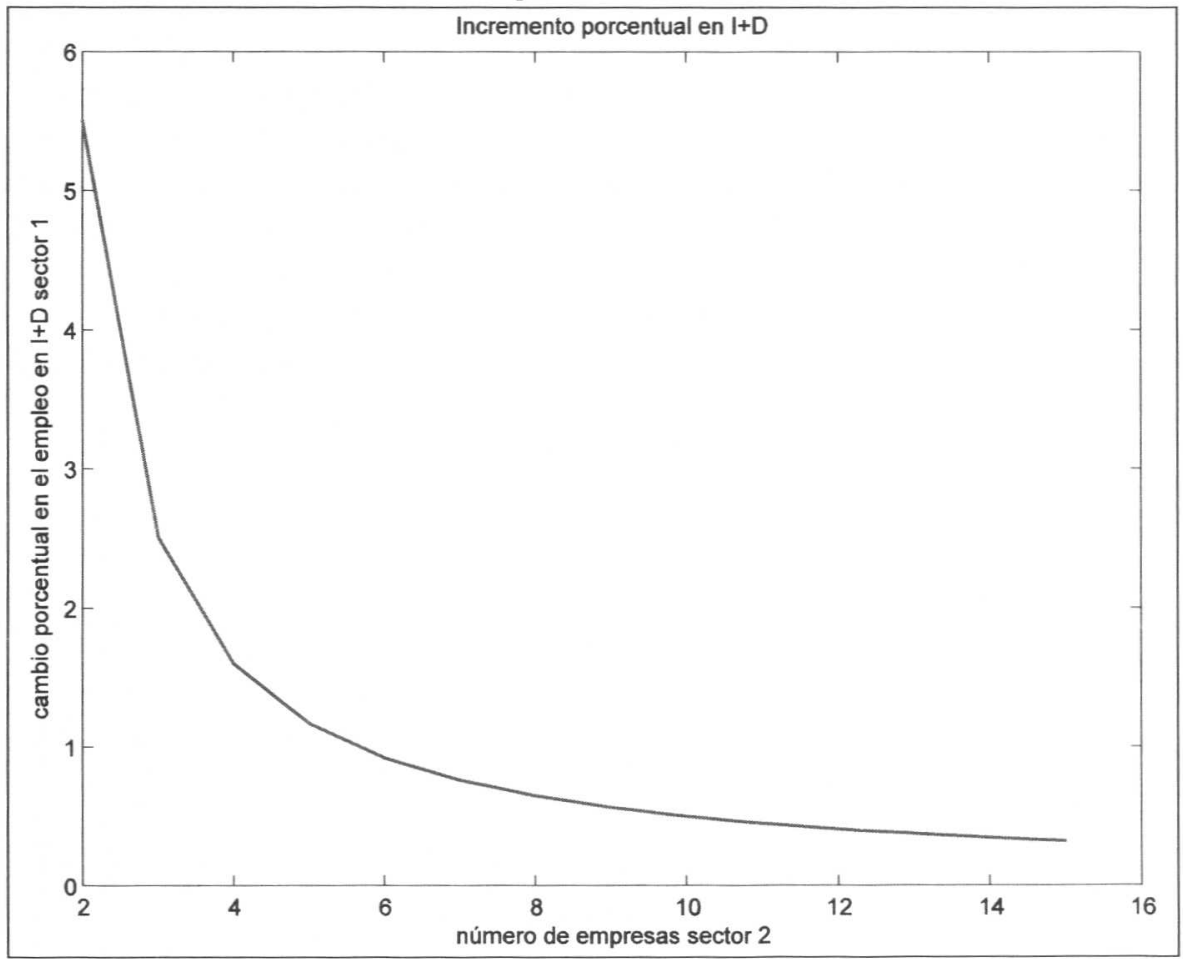

Para responder a la pregunta sobre que sectores son los que en un principio aumentan más su innovación como consecuencia de la apertura a los mercados internacionales, lo que vamos a hacer es estudiar la variación porcentual en el empleo en investigación y desarrollo tras la apertura, manteniendo constante el número de empresas de un sector y haciendo crecer el número de empresas del otro sector en autarquía. 
Figura 2.--Innovación en el sector 1 en presencia de diferencias sectoriales

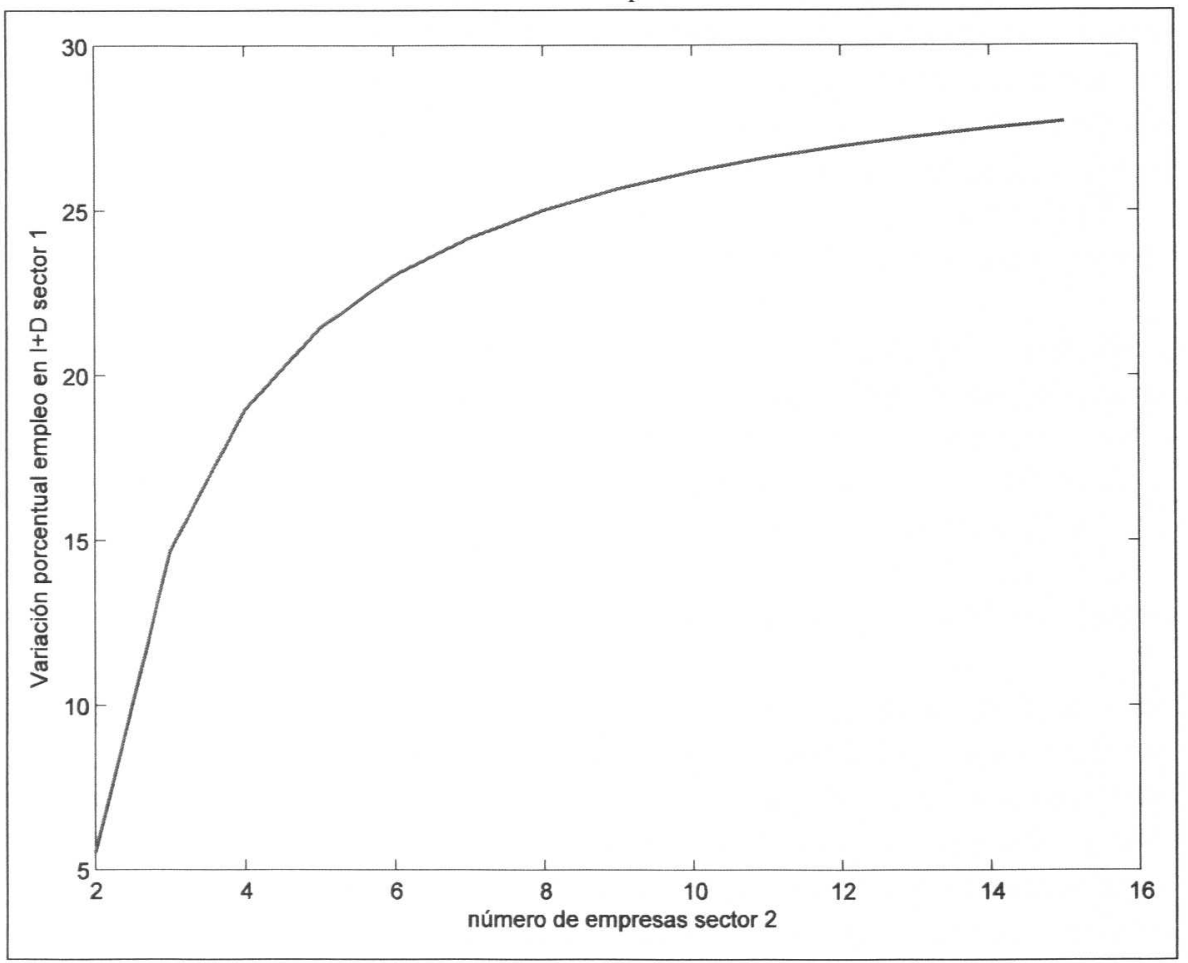

La figura 2 muestra claramente qué ocurre cuando abrimos la economía a los mercados internacionales en un contexto de diferencias de competencia a nivel intersectorial. En el gráfico, hemos considerado que el número de empresas en el sector 1 se mantiene fijo en dos, mientras que en el eje de abscisas encontramos el número de empresas en el sector 2, ambos en autarquía. En el eje de ordenadas nos encontramos con la variación porcentual del empleo en investigación y desarrollo por empresa que experimenta el sector 1 cuando la economía se abre al comercio internacional. Vemos que a medida que el sector 2 es más competitivo, el aumento en el empleo en investigación y desarrollo que experimenta el sector 1 es mucho mayor. La variación puede ser importante, de un 5\% cuando el número de empresas es el mismo e igual a 2 en autarquía, a un $25 \%$ aproximadamente cuando el número de empresas en el sector 2 en autarquía es mayor de 10.

Es fácil trasladar este gráfico a un entorno de dos países con diferencias sectoriales en niveles de competencia (diferencias entre sectores pero no diferencias entre países). En aquellos 
sectores caracterizados por bajos niveles de competencia, la apertura a los mercados internacionales produce aumentos considerables en los esfuerzos a innovar de las empresas.

Nos queda por saber qué ocurre con la inversión en I+D del sector 2 a medida que este es más competitivo inicialmente. La figura 3 nos muestra que efectivamente a medida que el sector 2 es más competitivo inicialmente menor es el aumento de los esfuerzos a innovar por empresa como consecuencia de la apertura a los mercados internacionales.

Observando la figura 3 además podemos darnos cuenta de que en realidad, la apertura a los mercados internacionales en presencia de diferencias sectoriales, reduce la inversión en innovación en ese sector. ${ }^{12}$

FIGURA 3.--Innovación en el sector 2 en presencia de diferencias intersectoriales

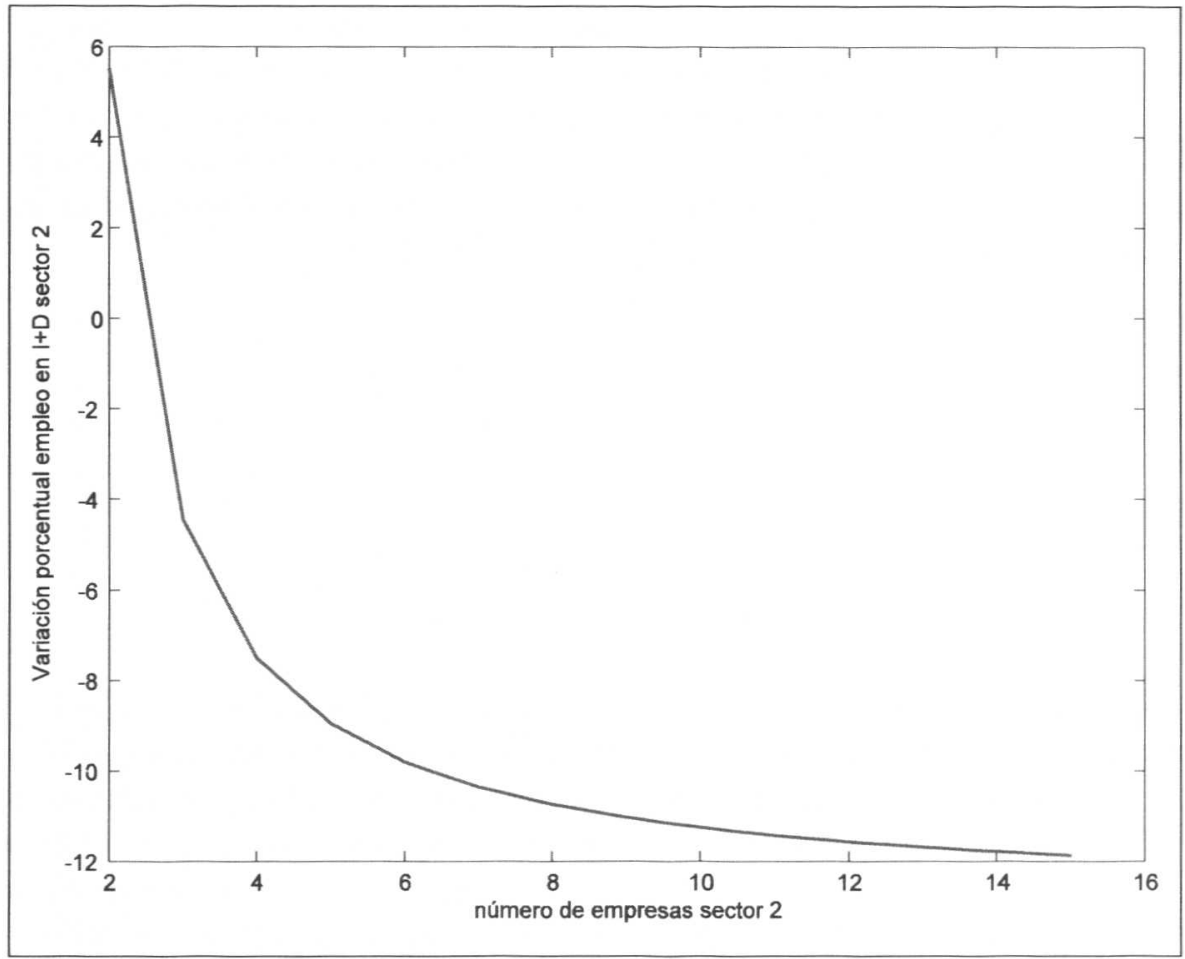

12 En el análisis de robustez, hemos encontrado que para otras configuraciones de los parámetros, la inversión de $\mathrm{I}+\mathrm{D}$ en el sector 2 (más competitivo) claramente disminuía en presencia de diferencias intersectoriales, pero era positiva si esas diferencias no eran muy grandes. 


\subsection{Liberalización de un único sector}

Para la realización de este ejercicio, hemos considerado únicamente una situación en donde no existen diferencias sectoriales inicialmente, es decir, consideramos que inicialmente los dos sectores tienen el mismo número de empresas. Esto se hace sin pérdida de generalidad, ya que el resultado no variará cualitativamente si permitimos diferencias sectoriales.

Los resultados se muestran en la figura 4. En el eje de abscisas tenemos el número de empresas que inicialmente existe en cada sector mientras que en el eje de ordenadas tenemos el empleo en $\mathrm{I}+\mathrm{D}$ del sector que no se abre al comercio (sector 2). Como era de esperar los esfuerzos en investigación y desarrollo caen en el sector que no se liberaliza. Vemos, no obstante, que en este caso la variación porcentual es mayor cuanto menos competitivos son ambos sectores. Esto es lógico, ya que conforme los dos sectores van siendo menos competitivos inicialmente, la ganancia en términos de competitividad del sector que se abre al comercio, el sector 1, es mucho mayor, luego el efecto crowding-out sobre el sector 2 es mucho mayor. 
Figura 4.--Innovación en el sector 2. Liberalización únicamente del sector 1

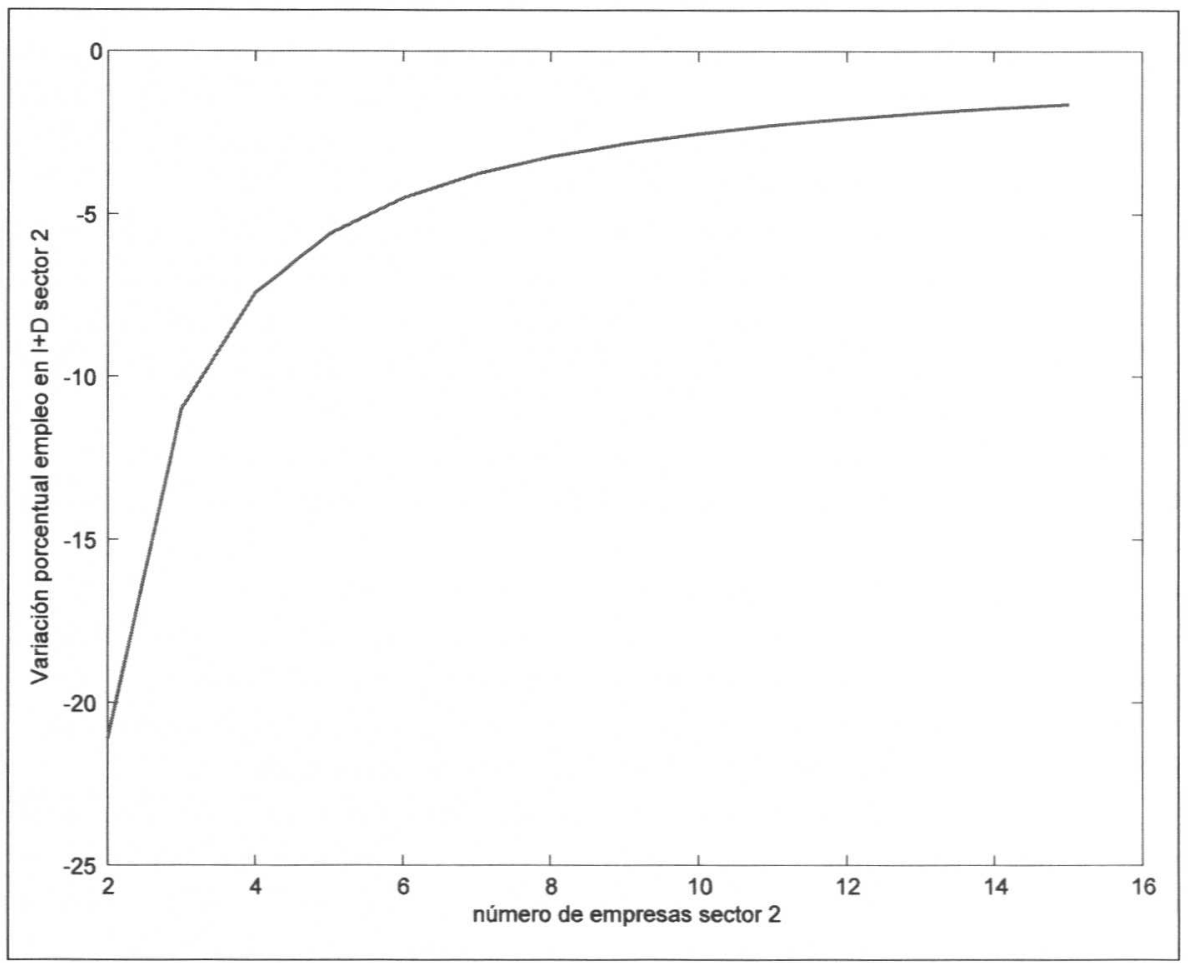

\section{CONCLUSIONES}

En este artículo hemos analizado cuáles son los efectos sobre la productividad a nivel de empresa que tiene la apertura al comercio entre dos economías simétricas donde existen diferencias sectoriales en niveles de competencia. Para ello hemos utilizado un modelo de competencia oligopolística donde cada variedad es producida por $n_{-}\{i\}$ empresas que compiten a la Cournot.

Hemos concluido que la apertura al comercio generará efectos distintos en sectores que presentan diferentes grados de competencia inicial. Los sectores menos competitivos son los que finalmente aumentarán más su volumen de producción y sus esfuerzos a la investigación como consecuencia de la apertura. Esto se debe a la existencia de un efecto no lineal en el modelo de Cournot: Cuanto menor es el nivel inicial de competencia, ma- 
yor es el impacto que la apertura al comercio internacional tiene sobre la elasticidad percibida de demanda. Este efecto provoca un aumento conjunto del volumen de producción total y de los esfuerzos dedicados a la innovación por empresa. En el caso límite, cuando los mercados se aproximan a competencia perfecta, el aumento en competencia es nulo y por tanto nulo es, el aumento en personal en investigación y desarrollo.

Además hemos descubierto que la apertura al comercio internacional, en presencia de diferencias intersectoriales, reduce el volumen de producción y la inversión en $\mathrm{I}+\mathrm{D}$ en el sector más competitivo. Esto es el resultado de una ganancia en eficiencia del sector 1 (debido a que aumenta su competencia más que el sector 2) que produce un efecto crowding-out sobre los factores de producción del resto de sectores.

El modelo es fácilmente extendible en varias dimensiones, las cuales serán acometidas en breve. Se puede considerar la introducción de diferencias sectoriales en otras dimensiones, tales como: peso de cada variedad en la función de utilidad (sería una proxy para un mercado doméstico mayor), preferencias CES a nivel más desagregado, para incluir diferencias sectoriales en la elasticidad de sustitución entre distintas variedades (aunque renunciando a algunas propiedades actuales del modelo), y experimentos de política comercial tales como, reducción simétrica de barreras arancelarias, reducción asimétrica (distinta entre sectores) de barreras arancelarias, etc..

Debemos interpretar no obstante los resultados obtenidos como efectos de medio plazo, donde el número de empresas permanece fijo y es exógeno. En una futura extensión del presente trabajo, introduciremos también endogeneidad en el número de empresas para tener un análisis más completo. En este ejercicio, la apertura al comercio generará una reducción en el número de empresas que opera en cada uno de los mercados, ya que el aumento de la competencia conllevará un descenso del margen de cada empresa y por tanto de los beneficios de cada empresa. En ese caso nos encontramos con un efecto del tipo selección: algunas empresas deben salir como consecuencia de la liberalización del comercio y esos recursos serán asignados a las empresas que quedan, aumentando su volumen de producción y sus esfuerzos a innovar.

\section{REFERENCIAS}

Aghion, P.; Harris, C.; Howitt, P. y Vickers, J. (2001), «Competition, Imitation and Growth with Step-by-Step Innovation», Review of Economic Studies, 68(3), págs. 467-492.

AtKeson, A. y Burstein, A. (2010), Innovation, Firm Dynamics, and International Trade, mimeo.

Bernard, A.; Eaton, J.; Jensen, J. B. y Kortum, S. (2003), «Plants and Productivity in International Trade», American Economic Review, 93 (4), págs. 1268-1290. 
BRANDER, J. (1981), «Intra-industry Trade in Identical Commodities», Journal of International Economics, 11(1), 1-14.

Brander, J. y Krugman, P. (1983), «A Reciprocal Dumping' Model of International Trade», Journal of International Economics, 15(3-4), págs. 313-321.

Bernhofen, D. (1999), «Intra-industry Trade and Strategic Interaction: Theory and Evidence», Journal of International Economics, 47(1), págs. 225-244».

Bloom, N.; Draca, M. y Van Reenen, J. (2008), Trade Induced Technical Change: The Impact of Chinese Imports on IT and Innovation, mimeo.

Bugamelli, M.; Fabiani, S. y Sette, E. (2008), The Pro-competitive Effect of Imports from China: An Analysis on Firm-level Price Data, mimeo.

Bustos, P. (2007), Trade Liberalization, Exports and Technology Upgrading: Evidence on the Impact of MERCOSUR on Argentinean Firms, mimeo.

Cellini, R. y LAmbertini, L. (2004), $R \& D$ Incentives and Market Structure: A Dynamic Analysis, mimeo.

Chen, N.; Imbs, J. y ScotT, A. (2009), «The Dynamics of Trade and Competition», Journal of International Economics, 77(1), págs. 50-62.

Devereux, M. y Lapham, B. (1994), «The Stability of Economic Integration and Endogenous Growth», The Quarterly Journal of Economics, 109(1), págs. 299-305.

Ederington, J. y McCalman, P. (2007), The Impact of Trade Liberalization on Productivity Within and across Industries: Theory and Evidence, mimeo.

Eslava, M.; Kugler, M. y Haltiwanger, J. (2009), «Trade Reforms and Market Selection: Evidence from Manufacturing Plants in Colombia», NBER WP 14935.

Fershtman, C. y Muller, E. (1984), «Capital Accumulation Games of Infinite Duration», Journal of Economic Theory, 33(2), págs. 322-339.

Fershtman, C. (1987), «Identification of Classes of Differential Games for Which the OpenLoop is a degenerated Feedback Nash Equilibrium», Journal of Optimization Theory and Applications, 55(2), págs. 217-31.

Grossman, G. y Helpman, E. (1991), Innovation and Growth in the Global Economy, The MIT Press.

Griffith, R.; Harrison, R. y Simpson, H. (2006), «The Link Between Product Market Reform, Innovation and EU Macroeconomic Performance», European Economy Economic Papers, 243.

Jones, C. (2002), «Introduction to Economic Growth», WW. Norton Univ. Press.

Impulliti, G. y Licandro, O. (2009), Trade, Firm Selection, and Innovation: The Competition Channel, mimeo.

Koeninger, W. y Licandro, O. (2006), «On the Use of Substitutability as a Measure of Competition», BE Topics on Macroeconomics, 6(6). 
Licandro, O. y Navas, A. (2010), «Trade Liberalization, Competition and Growth», Working Papers Instituto de Análisis Eco. (IAE).

Lileeva, A. y Trefler, D. (2007), «Improved Access to Foreign Markets Raises Plant-Level Productivity for Some Plants», NBER WP 13297.

Melitz, M. (2003), «The Impact of Trade on Intra-Industry Reallocation and Aggregate Industry Productivity», Econometrica, 71(6), págs. 1695-1725.

Melitz, M. y Ottaviano, G. (2008), «Market Size, Trade and Productivity», Review of Economic Studies, 75(1), 295-316.

Navas, A y Sala, D. (2007), «Technology Adoption and the Selection Effect of Trade», EUI ECO 20017/58.

NEARY, P. (2009), International Trade in General Oligopolistic Equilibrium, mimeo.

Peretto, P. (1999), «Cost Reduction, Entry, and the Interdependence of Market Structure and Economic Growth», Journal of Monetary Economics, 43(1), págs. 173-195.

- (2003), «Endogenous Market Structure, and the Growth and Welfare Effects of Economic Integration», Journal of International Economics, 60(1), págs. 177-201.

Reinganum, J. (1982), «A Class of Differential Games for Which the Closed Loop and Open Loop Nash Equilibria Coincide», Journal of Optimization Theory and Applications, 36(2), págs. 253-262.

Rivera-Batiz, L. y Romer, P. (1991), «Economic Integration and Endogenous Growth», The Quarterly Journal of Economics, 106(2), págs. 531-556.

Romer, P. (1990), «Endogenous Technological Change», Journal of Political Economy, 98(5), págs. 71-102.

TraCA, D. (2002), «Imports as Competitive Discipline: The Role of the Productivity Gap», Journal of Development Economics, 69(1), págs. 1-21.

VIVES (2008), «Innovation and competitive pressure» Journal of Industrial Economics, vol. 56, núm. 3 págs. 419-469. 


\section{APÉNDICE}

Derivación general del modelo de Cournot:

El problema general consiste en maximizar:

$$
\begin{gathered}
\operatorname{Max}\left(P(Q)-c_{i}\right) q_{i} \\
\text { s.a. } Q=\sum_{i=1}^{n_{i}} q_{i}
\end{gathered}
$$

La condición de primer orden asociada a este problema viene dada por:

$$
P(Q)+P(Q) q_{i}=c_{i}
$$

Donde los efectos que hemos comentado anteriormente en el texto corresponden a los términos primero y segundo de la parte izqda de la ecuación. Dividiendo por $\mathrm{P}(\mathrm{Q})$ a ambos lados y multiplicando y diviendo el segundo miembro por $Q$ tenemos que:

$$
1+\frac{\mathrm{P}(\mathrm{Q})}{P(Q)} Q \frac{q_{i}}{Q}=\frac{c_{i}}{P(Q)}
$$

donde fijémonos que podemos expresar esta condición como:

$$
1-\frac{s_{i}}{\varepsilon_{D}}=\frac{c_{i}}{P(Q)}
$$

donde la parte izquierda es $\theta_{i}$ que depende de $\frac{s_{i}}{\varepsilon_{D}}$ que es la inversa de la elasticidad percibida de demanda. 